University at Albany, State University of New York

Scholars Archive

$12-2014$

\title{
Epistemic categories and causal kinds
}

\author{
P.D. Magnus \\ University at Albany, State University of New York, pmagnus@albany.edu
}

Follow this and additional works at: https://scholarsarchive.library.albany.edu/cas_philosophy_scholar

Part of the Epistemology Commons

\section{Recommended Citation}

Magnus, P.D., "Epistemic categories and causal kinds" (2014). Philosophy Faculty Scholarship. 22.

https://scholarsarchive.library.albany.edu/cas_philosophy_scholar/22

This Review is brought to you for free and open access by the Philosophy at Scholars Archive. It has been accepted for inclusion in Philosophy Faculty Scholarship by an authorized administrator of Scholars Archive. For more information, please contact scholarsarchive@albany.edu. 


\section{Essay review}

Epistemic categories and causal kinds

P.D. Magnus

Department of Philosophy

University at Albany, State University of New York

Humanities 257

Albany, New York 12222

USA

\section{Natural categories and human kinds: Classification in the natural and social sciences.}

Muhammad Ali Khalidi, Cambridge University Press, 2013, pp. 264, Price $£ 55$ Hardcover, ISBN: 9781107012745

Within philosophy of science, debates about realism often turn on whether posited entities exist or whether scientific claims are true. Natural kinds tend to be investigated by philosophers of language or metaphysicians, for whom semantic or ontological considerations can overshadow scientific ones. Since science crucially involves dividing the world up into categories of things, however, issues concerning classification ought to be central for philosophy of science. Muhammad Ali Khalidi's book fills that gap, and I commend it to readers with an interest in scientific taxonomy and natural kinds. He works through general issues to craft a useful philosophical conception and uses the account to think through a wide range of specific examples. ${ }^{1}$

Although there are differences in the details, that one-sentence summary of Khalidi's book could just as well describe my own recent monograph on natural kinds (Magnus 2012). Since mine was published just shortly before Kahlidi's. neither of us was able to respond to the other. I regret this, because I would have profited from considering his view while developing my own. We both argue that natural kinds are discoverable by science and that a philosophical account of natural kinds must be able to make sense of substantive scientific cases. Examples, in their details, do as much work as general principles. We also agree that understanding natural kinds requires considering the causal patterns in the world that maintain

${ }^{1}$ Of course, there are different things one might want out of a theory of natural kinds. If one begins with an essentialist agenda, then one might say that what Khalidi would identify as 'natural kinds' are not really natural kinds at all. One would be forced into either nihilism about natural kinds (there are none) or a spartan fundamentalism (e.g., the only natural kinds are the posits of fundamental physics). Khalidi's response, given a prominent place in the last lines of his book, is that he can allow such a critic to have the phrase 'natural kind'. The phrase itself is not important. Khalidi wants to understand what distinguishes the categories identified by various sciences from arbitrary and frivolous ones. 
them, although we disagree as to whether causation can figure in defining what it is to be a natural kind. That disagreement must be understood against a background of considerable agreement. The critical comments which follow my brief summary should be accepted in that spirit and should not eclipse my recommendation of this book as one of the best on the topic of natural kinds.

In Chapter 1, Khalidi argues against overly metaphysical conceptions of natural kinds. The main target here is essentialism, which holds that a natural kind corresponds to a set of necessary and sufficient conditions specifying intrinsic, microphysical properties, which kind members possess in all possible worlds. He argues that essentialists ask too much. The tenets of essentialism are neither satisfied by scientific categories which seem prima facie to be natural kinds nor sufficiently motivated on independent grounds. Although a natural kind cannot simply be marked by a disjunctive hodgepodge of properties, it can be characterized by clusters or structures of properties which are not all possessed by all members. Furthermore, the properties themselves can have vague boundaries.

Rather than imposing a priori metaphysical constraints on the concept of a natural kind and then looking to science, Khalidi argues that there are a number of independent and defeasible constraints: the categories which are accepted to be exemplary natural kinds, our prior philosophical accounts, and the practice and results of actual empirical science. These cannot all be reconciled without at least some revision, and Khalidi recommends that we aim for reflective equilibrium revise as needed until our separate commitments can be reconciled.

In Chapter 2, Khalidi develops his positive account of natural kinds in relation to prior accounts given by other philosophers. He first provides an epistemic account of natural kinds according to which natural kinds are projectible categories, the ones which "enter into empirical generalizations, summarize a wealth of data, feature in explanations, give rise to valid predictions, and so on" (p. 54). He takes this epistemic conception to ground a positive presumption that categories which appear in our scientific accounts of the world correspond to natural kinds. Khalidi is a fallibilist and recognizes that all of the findings of our science are provisional and revisable, but he thinks that any failures of present scientific categories to track natural kinds are mistakes that can be corrected by further enquiry. Khalidi writes that "in the absence of specific claims to the contrary regarding actual scientific categories, we can take science to aim... at discovering the kinds of things that actually exist, and we can conclude that, unless there is a specific reason to regard a scientific category as arbitrary or biased, scientific categories are discovered rather than merely invented and will correspond to natural kinds" (p. 46).

Khalidi worries that this conception of natural kinds is subject to a sort of Euthyphro problem: a kind is discoverable by science because it is a natural kind, so its status as a natural kind cannot be $d u e$ to its being discoverable by science. He resolves the problem by arguing that the cluster of properties which are characteristic of a natural kind are held together by real causal patterns - that is, a pattern of underlying causes is what explains why the properties obtain together the way they do. So he suggests a "simple causal theory" of natural kinds according to which "a natural kind is associated with a set of properties whose co-instantiation 
causes the instantiation of other properties" (p. 80). ${ }^{2}$ He concludes, "Natural kinds of entities are nodes in causal networks" (p. 200).

The epistemic conception of natural kinds leads to the causal conception, and it is the causal conception on which Khalidi ultimately hangs his hat. The epistemic conception is not taken to define or constitutively distinguish natural kinds. Nevertheless, discoverability by science remains as an indicator of real causal patterns that might constitute a kind. Projectibility remains as "a diagnostic feature of natural kinds" (p. 98).

In Chapters 3 and 4, Khalidi argues that there are natural kinds, not just at the most fundamental level, but also in the special sciences. He argues that a natural kind is relative to a scale of size, a scale of time, and specific aspects of the objects and phenomena at those scales - that is, relative to a domain. Although this is a domain of enquiry, it is not constructed by enquiry itself. Instead, it is a part of the world and the natural kind is a real causal pattern discovered by science. He cleverly begins in Chapter 3 with fluid dynamics as his specimen. Although it is a precise and formal branch of physics, it is a special science in the sense that it neither applies to everything everywhere nor is it about microphysical fundamentals. Khalidi argues that there are natural kinds in fluid dynamics (like Newtonian fluid) and so shows that there are natural kinds in special sciences without becoming embrangled in complications related to biological and social phenomena. He turns to those complications in Chapter 4, arguing that historical and interactive kinds can be natural kinds.

In Chapter 5, Khalidi engages a succession of specific examples: lithium, polymer, virus, cancer, and attention-deficit disorder. In each case the discussion depends, not just on one would-be kind in isolation, but instead on broader taxonomic considerations from that domain of science. To consider just one case, Khalidi argues that a chemical element has the necessary condition of a specific atomic number (3, in the case of lithium) but only a disjunctive array of properties which are associated with its various isotopes. In most cases, each isotope will also be a natural kind, and other natural kinds might take in specific isotopes along with isotopes of other elements; for example, lithium-8 and beryllium-8 can be categorized together as beta-minus decay nuclides. The other case studies are similarly responsive to the details of actual science. Each of them is rewarding for a reader interested in a naturalistic account of natural kinds, independently of the fine details of Khalidi's own conception.

In Chapter 6, Khalidi deals with the general question of whether natural kinds in his sense are real. It is traditional to understand reality in terms of mindindependence, but Khalidi argues that this is unhelpful. Although kinds of mental events are trivially mind-dependent, Khalidi's defense of special science kinds allows that psychological categories of mental events can correspond to real natural kinds. Their banal mind-dependence as features of mind is no mark against their reality, and it proves difficult to distinguish that from a variety of mind-dependence which is. So Khalidi suggests that natural kinds are real unless they are subjective,

2 Khalidi takes the phrase "simple causal theory" from Craver 2009; see e.g. Khalidi 2013, p. 78. 
and "what we want to avoid is subjectivity in the sense of biases that subvert our epistemic purposes" (p. 222). One might worry that the Euthyphro problem returns here. The reality of natural kinds is characterized in terms of our epistemic purposes, one of which is to discover the kinds that exist. Yet here, as earlier, Khalidi suggests that epistemic considerations can be cashed in for causes. He formulates "realism about natural kinds" in this way: "Natural kinds divide the world into individuals that share causal properties, enter into the same or similar causal relationships, and give rise to the same or similar causal processes" (p. 222). So he takes realism about natural kinds to coincide with the causal account that he articulates and defends.

Note that Khalidi's account does not treat natural kind itself as a strict category with sharp boundaries. The properties characteristic of a kind can hold together with a degree of stability across a range of circumstances and obtain more or less often in the actual world, so kinds might count as more or less natural. This 'more or less' is not even a matter of degree, strictly speaking, because the contrast between natural and non-natural kinds does not occur along just one dimension. Kinds can differ by the range and number of associated properties, by the strength of the association, by the background conditions which must obtain in order for the association to hold, by the simplicity or complexity of the causal network connecting those properties, and by their prevalence in the world (pp. 212-4). So a kind might be more natural than another in some of these respects even while the second is more natural than the first in others. Just as he rejects essentialism about natural kinds, Khalidi denies essentialism about the category natural kind itself. This does not erode the usefulness of the category. It often suffices in specific cases to determine which of several candidate kinds is more natural, and there are still some kinds which are not natural kinds even a little bit.

Moreover, the slipperiness of degrees of naturalness can be reduced by recalling that natural kinds are relative to domains. The kind beta-minus decay nuclide only makes sense and so can only be natural in a domain which includes beta decay processes. Yet - and here I switch from summary to critical engagement - there are two different ways of understanding this domain-dependence. First, one might suppose that it is possible to identify natural kinds without mentioning domains but that every natural kind so-identified occupies some domain or other. Second, one might think that a category's status as a natural kind is a relation between a kind and a domain. The former presumes what I call the simpliciter assumption, that ' $\mathrm{K}$ is a natural kind' is a complete thought. The latter holds that the very concept of natural kind incorporates domain-relativity. There is always at least an implicit domain, and the complete thought is ' $\mathrm{K}$ is a natural kind for domain D.' I explicitly reject the simplicter assumption and adopt the latter approach. ${ }^{3}$

Although Khalidi argues that natural kinds hold in domains, he does not include domains explicitly in his definition of what it is to be a natural kind. In this sense, his account resembles the one given by Anjan Chakravartty, who views a natural kind as an array of causal properties that tend to occur together. Adopting a metaphor from Bertrand Russell, Chakravartty describes the properties as sociable,

3 See Magnus 2012, pp. 39-43; see also Boyd 1999, p.148.\} 
reflecting "the fact that some collections of property instances like each other's company and others do not" (Chakravartty 2007, p. 170). As he notes, the view is "somewhat deflationary about kinds themselves" (Chakravartty 2007, p. 178). ${ }^{4} \mathrm{~A}$ problem with this approach is that similarity is ubiquitous. Any arbitrary collection of things is similar in some respects, and any bundle of properties is sociable to some degree. If collections were well-ordered with respect to their degree of sociability, we might just accept that natural kindness comes in degrees. However, as we've seen, kinds can be more or less natural in indefinitely many respects. So I worry that there will rarely or perhaps even never be an answer to which of two property bundles are more sociable simpliciter (cf. Magnus 2012, pp. 127-9). Rejecting the simpliciter assumption mitigates this worry, because specifying a domain picks out certain aspects of similarity as the salient or important ones. The domain-relative conception of natural kind might still be a matter of degree, but the range of relevant respects is constrained by the domain itself. As Khalidi writes, "different categories may be found to be important from the perspective of different scientific disciplines" (p. 54).

Khalidi's account, it seems to me, is independent of the simpliciter assumption. It can be interpreted to be more like Chakravartty's or more like mine. His slogan that to be a natural kind is to be a node in a causal network can be read in two ways. In one way, it claims that ' $\mathrm{K}$ is a natural kind' means 'there is a causal network in the world such that $\mathrm{K}$ is a node in it'. In the other, it claims that ' $\mathrm{K}$ is a natural kind in domain D' means 'D constitutes a causal network such that $\mathrm{K}$ is a node in D'. Since many but not all philosophers writing about natural kinds accept the simpliciter assumption, the flexibility of Khalidi's account is perhaps to his credit.

I am more concerned by the relation between Khalidi's epistemic and causal accounts of natural kinds. I would prefer to see the two accounts as answering somewhat different questions. The epistemic account, posed in terms of the categories which can support successful science, answers a question about which categories are natural kinds. The causal account answers the somewhat different question about what in the world holds those natural kinds together. I call these the taxonomy and ontology questions, respectively, and I think that it is important not to collapse them. ${ }^{5}$

These should be posed as separate questions because consideration of causes gives neither a complete nor uniform answer to the ontology question. Some kinds, like the particles of fundamental physics, are arguably not held together by causes at all - rather, their unity is just a basic feature of the universe. Even where causes do unify a kind, they can do so in two importantly different ways. All the members of a biological species are part of the same lineage, whereas the instances of a particular chemical compound might be entirely independent. For example: Every duck in the species Anas platyrynchos is a descendent of common ancestors, and they all figure in the same token causal history. Every pool of water is organized by the causal interaction between hydrogen and oxygen atoms, but two historically unconnected

${ }^{4}$ Khalidi cites Chakravartty approvingly (pp. 14,42).

${ }^{5}$ See Magnus 2014 and forthcoming. 
pools are distinct tokens of that type. There is an important difference between the former sort of token-causal kinds and the latter sort of type-causal kinds. ${ }^{6}$

Khalidi acknowledges these points, but draws different lessons from them than I do. Regarding the different ways that causes can hold a kind together, Khalidi is centrally concerned to argue that historical, etiological categories can be natural kinds. So, although he acknowledges a difference between kinds in which all members share a common history and kinds which allow for independent histories, he is concerned primarily to underscore the similarities between the two (pp. 1307 ). The difference between token-causal and type-causal kinds does not mark the difference between kinds in biological sciences and kinds in physical sciences; although species are token-causal, many biological kinds are not. Nor does it mark the difference between ersatz and properly natural kinds; both are ways that natural kinds can be realized. Yet there is an important difference between them metaphysically; token-causal kinds can be conceptualized as historically continuous individuals, but type-causal kinds cannot. ${ }^{7}$

Regarding the possibility that microphysical kinds might be non-causal, Khalidi notes that physicists are not all in agreement about the absence of causes at the fundamental level and that perhaps future discoveries will overthrow our present understanding. This is not something to be settled in advance on the basis of philosophical scruples, but perhaps there just are no causes to be found among quarks. In that case, Khalidi writes, we could "posit two classes of natural kinds that constitute real features of the universe for different reasons or on different grounds" (p. 211). It seems to me, however, that even allowing such a possibility brings back the Euthyphro problem. The question is not just what would hold together a noncausal natural kind - the kind might just be grounded in brute features of the universe - but rather by what dint such a group would count as a natural kind at all. If the Euthyphro problem for the epistemic conception were serious, then the possibility that some natural kinds could turn out to be non-causal would be a disaster.

The resolution, I suggest, is that the problem is not really that serious. We can be realist about the features which support our epistemic access to the world even without specifying the details of how that status is realized. Any answer to the taxonomy question is already a little bit metaphysical.

Khalidi holds that discovering kinds is just part of the aim of science, and he accepts presumptively that categories which appear in present science correspond to natural kinds. He acknowledges that this presumption is defeasible, because future scientists will be forced to make some revisions. One wonders, however, why scientists should make any revisions if natural kinds are not somehow more than

${ }^{6}$ In my work, I pose the distinction in the context of the Homeostatic Property Cluster (HPC) account of kinds and call these token-HPCs and type-HPCs, respectively. Khalidi rejects the HPC approach in the course of arguing for his simple causal theory, but to some extent this is just a difference in labels. Whereas he takes criticisms as a reason to move beyond the HPC monicker, my reaction to similar criticisms is to offer a revised HPC account.

${ }^{7}$ For another application of this distinction, see Magnus 2013. 
epistemic. So the general presumption, that kinds in the natural science are natural kinds, raises something like the Euthyphro problem. This worry does not arise in the same way for my own account, because I do not accept a general presumption that present scientific categories correspond to natural kinds. I argue, for example, that most species taxa are natural kinds but that the species category itself is not (Magnus 2012, pp. 83-96, 172-175). We should think that the categories in science are natural kinds when epistemic success would only be possible with those categories. Beyond just success, this is a further question of whether other categories could have delivered comparable success. My characterization of natural kinds thus has two parts: the success clause requires that the category can figure in enquiry that yields inductive and explanatory success, and the restriction clause requires that the success would not be possible without that category. Roughly, Khalidi's epistemic conception just consists of the success clause. There are not natural kinds (on my account) if success could be attained with an indefinite number of radically different category schemes. Because natural kinds are indispensable for inductive and explanatory success in a domain where there are natural kinds, scientists pursue natural kinds because they pursue induction and explanation - not because aiming to discover the kind-structure of things is an independent aim. Natural kinds in this sense are constraints the world imposes on enquiry, i.e. real features of the world.

So the conjunction of the success and restriction clauses can give a freestanding answer to the question of which groups of things in the world form natural kinds. What there is in the world that holds natural kinds together is a separate and subsequent question. We are free to give different answers to that subsequent question for different kinds. Some are held together in virtue of similar causal patterns active in each instance of the kind. Some are the result of a common origin and constitute a kind because each member still carries the mark of that origin. Some might not be causal at all.

It is no surprise, in the end, that I disagree with Khalidi's positive account precisely where it disagrees with my own account. I reiterate, however, that this disagreement comes after considerable agreement. Khalidi addresses some of these at greater length than I do: Negative arguments against essentialist and nonnaturalist conceptions of natural kinds; Arguments that there is a unified conception of natural kinds which allows for there to be natural kinds in biological and social domains, rather than different conceptions for the physical sciences and biology or a conception so narrow as just to allow for microphysical kinds; And numerous case studies which show by nuanced argument that and how particular examples are natural kinds.

\section{References}

Boyd, R. (1999). Homeostasis, species, and higher taxa. In R.A. Wilson, editor, Species: New interdisciplinary essays. The MIT Press, Cambridge, Massachusetts: The MIT Press. 141-185.

Chakravartty, A. (2007). A metaphysics for scientific realism. Cambridge University Press. 
Craver, C.F. (2009). Mechanisms and natural kinds. Philosophical Psychology, 22(5):575-594, October.

Magnus, P.D. (2012) Scientific enquiry and natural kinds: From planets to mallards. Basingstoke, Hampshire: Palgrave MacMillan.

-, - . (2013). Historical individuals like Anas platyrhynchos and 'Classical gas'. In C. Mag Uidhir, editor, Art \& abstract objects. Oxford University Press. 108-124.

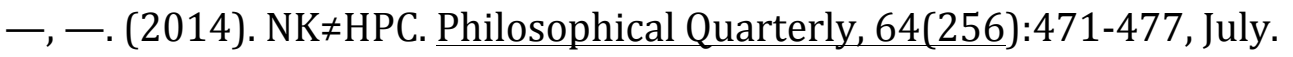

-, - . (forthcoming). John Stuart Mill on taxonomy and natural kinds. HOPOS: The Journal of the International Society for the History of Philosophy of Science. 